\title{
Development of Ground-Based Search-Coil Magnetometer for Near-Earth Space Research
}

\author{
Jehyuck Shin ${ }^{1}$, Khan-Hyuk Kim ${ }^{1,2}$, Ho Jin ${ }^{1,2 *}$, Hyomin Kim ${ }^{3}$, Jong-Woo Kwon ${ }^{1}$, Seungah Lee ${ }^{2}$, \\ Jung-Kyu Lee ${ }^{1}$, Seongwhan Lee ${ }^{1}$, Geonhwa Jee ${ }^{4}$, and Marc R. Lessard ${ }^{5}$ \\ ${ }^{1}$ School of Space Research, Kyung Hee University, Yongin 17104, Korea \\ ${ }^{2}$ Department of Astronomy and Space Science, Kyung Hee University, Yongin 17104, Korea \\ ${ }^{3}$ Center for Solar-Terrestrial Research, New Jersey Institute of Technology, Newark 07102, USA \\ ${ }^{4}$ Korea Polar Research Institute, Incheon 21990, Korea \\ ${ }^{5}$ Space Science Center, University of New Hampshire, Durham 03824, USA
}

(Received 21 October 2016, Received in final form 19 December 2016, Accepted 19 December 2016)

\begin{abstract}
We report on development of a ground-based bi-axial Search-Coil Magnetometer (SCM) designed to measure time-varying magnetic fields associated with magnetosphere-ionosphere coupling processes. The instrument provides two-axis magnetic field wave vector data in the Ultra Low Frequency or ULF (1 $\mathrm{mHz}$ to $5 \mathrm{~Hz})$ range. ULF waves are well known to play an important role in energy transport and loss in geospace. The SCM will primarily be used to observe generation and propagation of the subclass of ULF waves. The analog signals produced by the search-coil magnetic sensors are amplified and filtered over a specified frequency range via electronics. Data acquisition system digitizes data at 10 samples/s rate with 16-bit resolution. Test results show that the resolution of the magnetometer reaches $0.1 \mathrm{pT} / \sqrt{\mathrm{Hz}}$ at $1 \mathrm{~Hz}$, and demonstrate its satisfactory performance, detecting geomagnetic pulsations. This instrument is scheduled to be installed at the Korean Antarctic station, Jang Bogo, in the austral summer 2016-2017.
\end{abstract}

Keywords : magnetometer, search-coil magnetometer, magnetosphere, ionosphere, ULF waves

\section{Introduction}

Magnetometers are one of the widely used instruments for space research. Among various types of magnetometers, "fluxgate" type magnetometers are often used to measure DC magnetic fields while "search-coil (or induction)" type magnetometers (SCM hereinafter) AC fields (dB/dt). SCMs are typically bigger and heavier than the fluxgate types. In spite of the disadvantage of their size, SCMs are still indispensable because of their sensitivity and durability [1]. A crucial parameter of SCMs is sensitivity to measure the weak signal in the Earth's magnetic field [12]. SCMs have no upper limit of sensitivity range and especially they are better than fluxgate magnetometers above $1 \mathrm{~Hz}[1,6]$. The ground-based models of SCMs are in operation in various locations for space research. Example projects and institutes include Canadian Array

CThe Korean Magnetics Society. All rights reserved.

*Corresponding author: Tel: +82-31-201-3865

Fax: +82-31-201-3852, e-mail: benho@khu.ac.kr for Realtime Investigations of Magnetic Activity (CARISMA), British Antarctic Survey (BAS), Augsburg College and University of New Hampshire (UNH). This paper describes a ground-based SCM developed by Kyung Hee University (KHU) in collaboration with $\mathrm{UNH}$. The design is largely based on that of UNH whose systems are widely used in the Arctic and Antarctic regions. It will be installed at one of the Korean Antarctic stations for the study of ULF wave generation and propagation, which is the first development of such an instrument by a Korean institute for near-earth space research.

ULF waves play an important role in energy transfer between the magnetosphere and ionosphere, and provide key information about physical processes of the geospace environment [10]. They cover roughly $1 \mathrm{mHz}-5 \mathrm{~Hz}$ frequency range: the subclass of the ULF wave include Pc15 and Pi1-2 (see [5] for the subclass of ULF waves). Various wave types of the ULF band have different occurrence factors and plasma conditions. The magnetometer described in this paper is designed to measure Pc1-2 
waves and Pi1-2 waves which are typically associated with electromagnetic ion cyclotron (EMIC) waves [7] and substorm activities $[4,13]$, respectively. ULF waves transmitted from the terrestrial magnetosphere are typically observed at high-latitudes [11, 3]. In the austral summer 2016-2017, the SCM is scheduled to be installed at Jang Bogo, the new Korean research station in Antarctica run by Korea Polar Research Institute (KOPRI).

Section 2 describes the instrument design and system configuration and specification of the SCM. Performance test and field test results are shown in Section 3. In section 4 , we summarize the overall system development and installation planning at the station.

\section{System Design}

The SCM consists of two magnetic sensors with onboard pre-amplifiers, and a control box containing a main analog board and a data acquisition system (DAQ) (Fig. 1). The design of the magnetic sensors and analog electronics in this project are based on that of the magnetometers originally developed by $\mathrm{UNH}$. The $\mathrm{UNH}$ magneto-

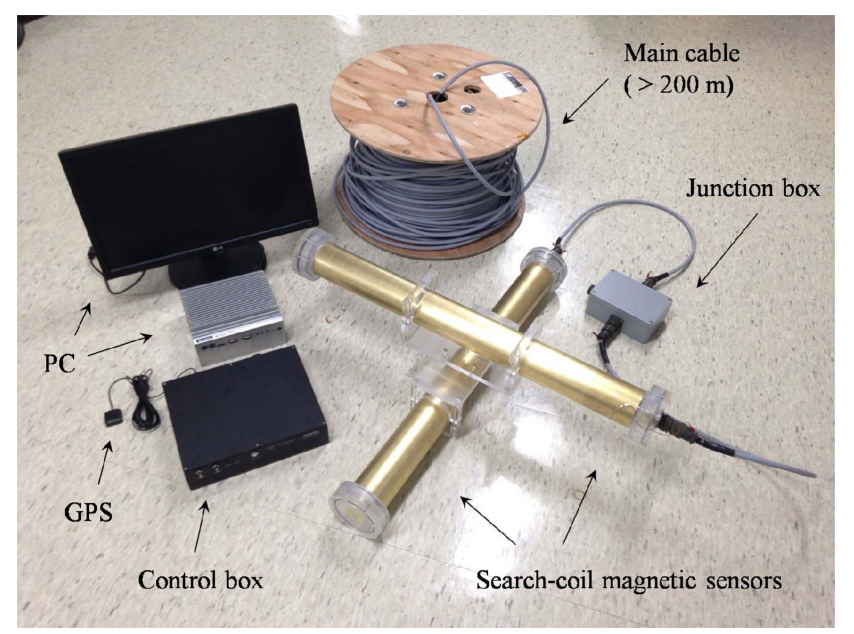

Fig. 1. (Color online) System configuration of the SCM. meters have long been used for ground-based observations of geomagnetic pulsations. The two search-coil magnetic sensors are identical and assembled orthogonally ( $\mathrm{X}$ and $\mathrm{Y}$ axis) using a sensor bracket: The $\mathrm{X}$ - and $\mathrm{Y}$-axis sensors are oriented along the geomagnetic north-south and east-west direction, respectively. The SCM system block diagram is shown in Fig. 2. Magnetic sensing signals detected by two axis sensors are amplified by the pre-amplifiers and transmitted to the main electronics through a $200-\mathrm{m}$ cable. The signals from each sensor are fed to the single main cable via a junction box located between the sensors and main electronics. The main analog board has a secondary amplifier and low pass filter to limit the frequency range of the detected signals. This filter has a cutoff frequency at $6 \mathrm{~Hz}$. The DAQ consists of data acquisition program and electronics which has a multi-channel Analog-to-Digital Converter (ADC). Acquired signals are sampled at the rate of 10 samples/s with time synchronization through the Network Time Protocol (NTP) and Global Positioning System (GPS) antenna/receiver. Digitized data are stored on a daily basis in a PC as ASCII files which are accessible via Network Attached Storage (NAS). The main analog board is equipped with an oscillator circuit to generate an artificial magnetic field signal from test coils inside each sensor to test the integrity of the magnetometer system. The magnetic sensors will

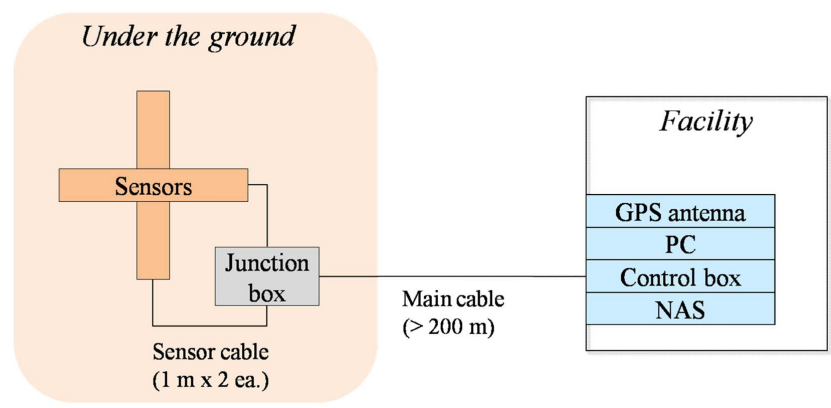

Fig. 3. (Color online) Concept diagram of the SCM installation.

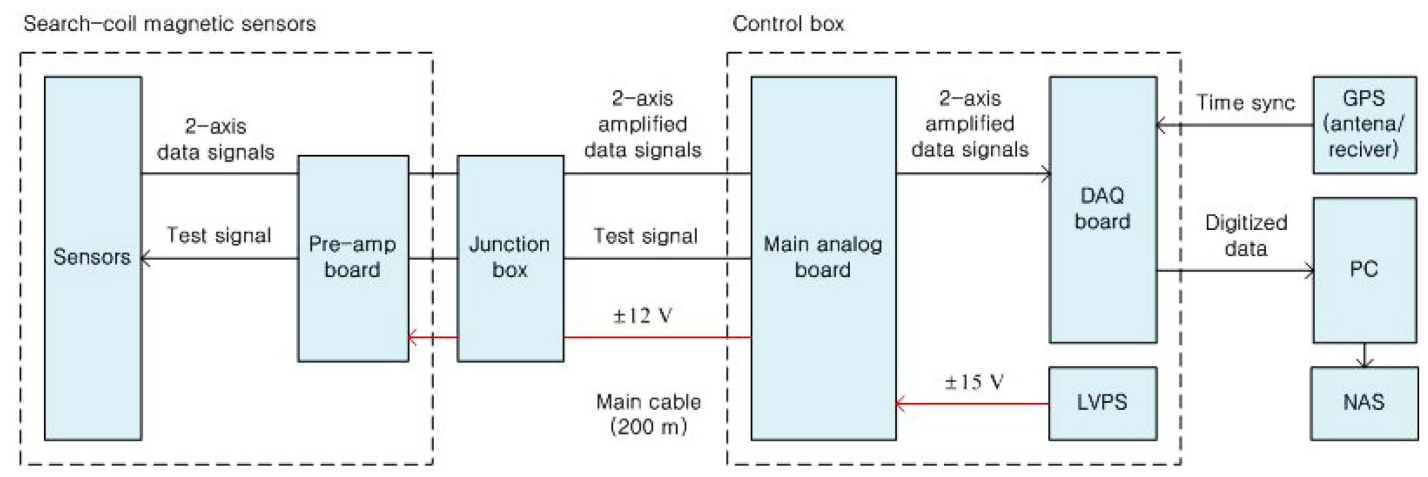

Fig. 2. (Color online) Block diagram of the SCM system. 
be installed under the ground or snow approximately 200 $\mathrm{m}$ away from the facility where the main electronics will be installed to minimize electromagnetic interferences (Fig. 3).

The sensor housing is designed to be durable for outdoor use (e.g., physical strength and waterproofness). The control box and computer will be located inside the station facility.

\subsection{Sensor}

The search-coil magnetic sensors are generally composed of enamel-coated copper wires wound around a high permeability $(\mu)$ ferromagnetic core to draw high voltage across a magnetic sensor coil $[8,9]$. To achieve appropriate amplification for our applications, hundreds of thousands coil turns are implemented on a core. Our coil spools are made of Acetal $\left(\right.$ Delrin $\left.^{\circledR}\right)$ which has no static discharge and high strength. Each spool has tens of thousands coil turns and dozens of spools were assembled to make up a single coil. The spools, core and pre-amplifier are housed in a polycarbonate tube (Fig. 4). Each sensor is 920 (L) $\times 130$ (D) $\mathrm{mm}$ in size and $10.5 \mathrm{~kg}$ in mass. The sensor housing is designed to be waterproof for outdoor use as described earlier and composed of easily disassemblable parts for easy repair (Fig. 5). The sensors also have a few tens of turns of wire to serve as a test coil also as described earlier.

The main purpose of the pre-amplifiers is to amplify signals before transmitting them to the main analog circuit via the long $(200 \mathrm{~m})$ main cable. The resonance frequency of the pre-amplifier combined with the coil is 4

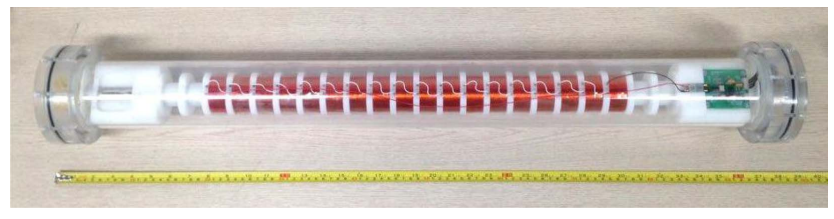

Fig. 4. (Color online) Search-coil magnetic sensor of the SCM.
Hz. The power for the pre-amplifier $( \pm 12 \mathrm{~V})$ is supplied from the main analog board via the main cable (Fig. 5, right).

\subsection{Main Analog Board}

The main analog board amplifies signals from the preamplifiers. There are three gain levels which will be adjusted to obtain magnetic field wave events of interest on a desired level. The resonance frequency is set approximately at $4 \mathrm{~Hz}$ (defined as $-3 \mathrm{~dB}$ from the maximum amplitude). The board also contains a circuit that generates an oscillating signal $\left(1 \mathrm{~Hz}, \mathrm{~V}_{\mathrm{pk}-\mathrm{pk}}\right)$ to generate a known magnetic field inside the magnetic sensor to test the integrity of the magnetometer system.

Power regulators are implemented to supply $\pm 12 \mathrm{~V}$ to the pre-amplifiers and main analog board, converting \pm 15 $\mathrm{V}$ from the linear power supply. The main analog board is $150 \times 130 \mathrm{~mm}$ in size, $88 \mathrm{~g}$ in mass and it is placed in the control box.

\subsection{Data Acquisition System}

Digitization and processing of data signals transmitted from the main analog board are performed in the DAQ. Our system employs the LabJack U6 model to serve as a multi-channel ADC which handles bi-axial data from the $\mathrm{X}$ and $\mathrm{Y}$-axis sensors. The DAQ board is powered by the USB port of an industrial computer. The DAQ is run by a program based on LabVIEW. The user interface is shown in Fig. 6. The sampling rate is 10 samples/s/axis, yielding 30.4 Mbyte/day for bi-axial data. A NTP and GPS antenna/receiver are used for accurate time synchronization with $1 \mathrm{~ms}$ time accuracy. Acquired data are saved automatically on the computer and transferred to a NAS in real time for public access.

\subsection{System Specification}

The specification of the SCM is shown in Table 1 which summarizes the technical details. The following section describes the tests performed.
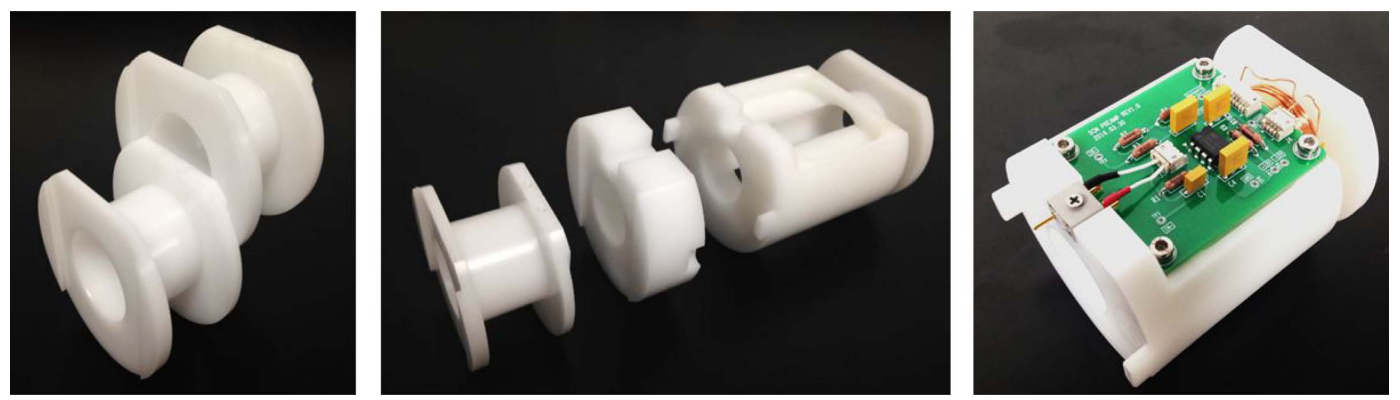

Fig. 5. (Color online) Spool and an on-board amplifier configuration. Each part has a slide fit design (left, middle). The pre-amp board is installed at the end of sensor configuration (right). 


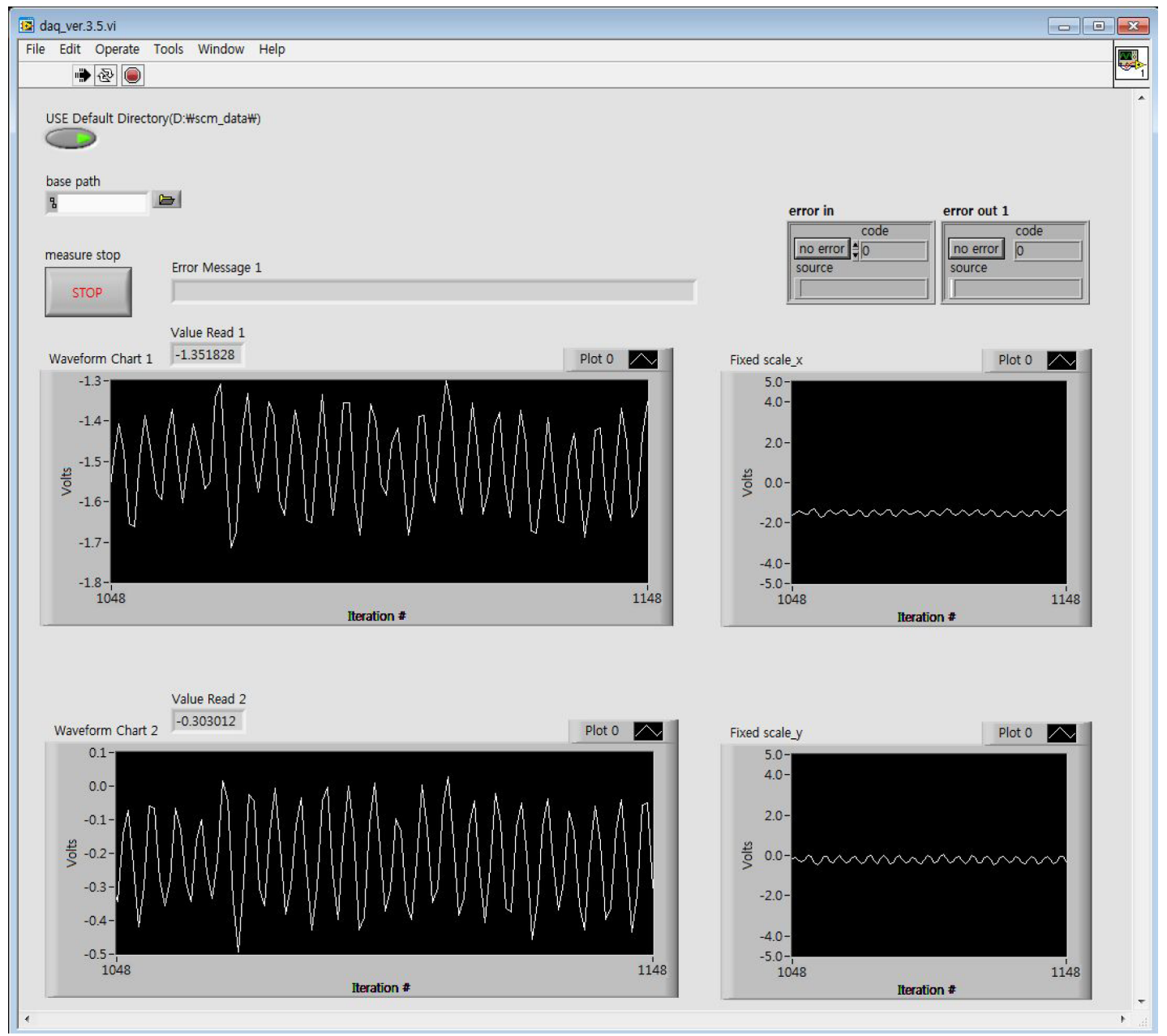

Fig. 6. (Color online) LabView-based user interface software.

Table 1. System Specification of the SCM.

\begin{tabular}{cc}
\hline \hline Type & Bi-axial search-coil magnetometer \\
\hline Frequency response & $1 \mathrm{mHz}$ to $5 \mathrm{~Hz}$ \\
Sensor sensitivity & $364 \mu \mathrm{V} /(\mathrm{nT} \cdot \mathrm{Hz})$ \\
Resolution & $0.1 \mathrm{pT/} \sqrt{\mathrm{Hz}} @ 1 \mathrm{~Hz}$ \\
Noise level & $0.07 \mathrm{pT} / \sqrt{\mathrm{Hz}} @ 1 \mathrm{~Hz}$ \\
Sampling rate & 10 samples/sec/axis \\
File size and format & 30.4 Mbyte/day $(2 \mathrm{ch})$, ASCII \\
\hline
\end{tabular}

\section{Performance Tests}

\subsection{Frequency Response}

A frequency response test is performed to characterize the resonance and cut-off frequency of the magnetometer $[2,14]$. An artificially generated external field using a test solenoid is used for this test. The solenoid has 360 turns of copper wire on a PVC pipe $(1300 \mathrm{~L} \times 155 \mathrm{D} \mathrm{mm})$ and is designed to provide magnetic signals around a single axis sensor. The setup for the test conducted in the Kyung

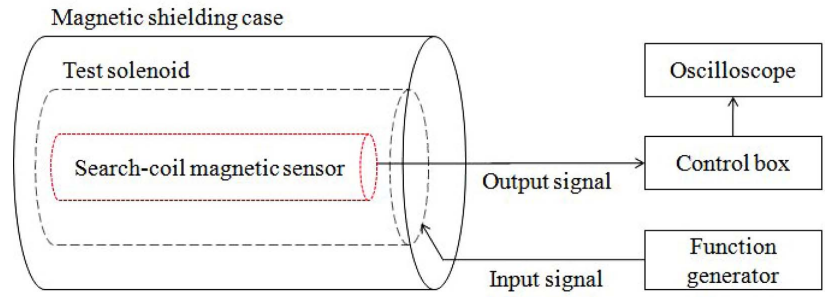

Fig. 7. (Color online) Test method for frequency response and resolution performance.

Hee University laboratory is shown in Fig. 7. To minimize electromagnetic interference, this test is carried out in a magnetic shielding case which is designed to block ambient magnetic field. A sinusoidal wave with a fixed amplitude using a function generator is applied to the test solenoid to create a time-varying magnetic field inside the solenoid. By sweeping the frequency $(0.1$ to $30 \mathrm{~Hz})$ of the test wave signal, the frequency response (output voltage amplitude versus test wave frequency) of the magnetometer is examined. The test result is shown in Fig. 8, 


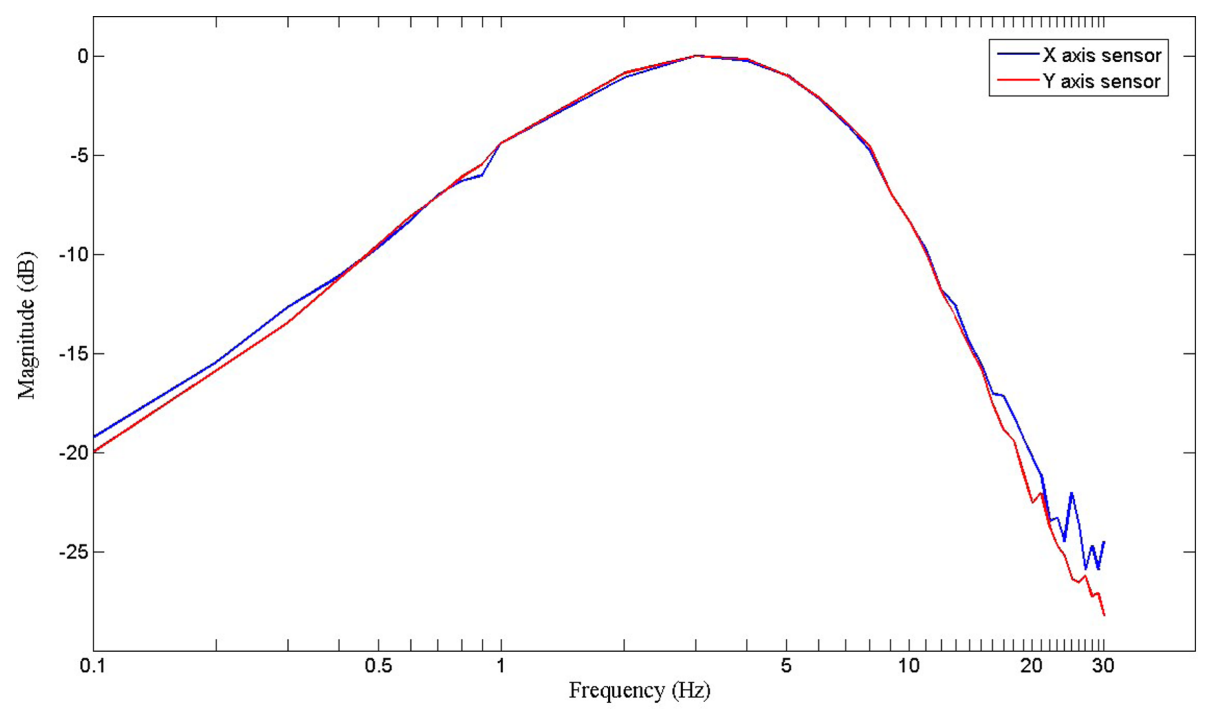

Fig. 8. (Color online) Frequency response curve. The resonance peak is shown near $\sim 4 \mathrm{~Hz}$.

showing the magnetometer's resonance frequency peaks at $\sim 4 \mathrm{~Hz}$ and its cutoff frequency at $\sim 6 \mathrm{~Hz}$ (defined as -3 $\mathrm{dB}$ corner frequency).

\subsection{Resolution}

A resolution of magnetometer, a measure of the smallest change it can detect, is one of the very important features defining the sensor performance. The noise level of the system also needs to be characterized to specify its resolution. This section describes how the sensor resolution and noise level are measured. This test is similar to the frequency test described in the previous section except that the input test signal amplitude is varied at a fixed frequency until the applied signal is only above $3 \mathrm{~dB}$ above the noise floor in a spectral domain (this is called "minimum discernible signal or MDS). This is measured by observing an output spectral peak using a spectrum analyzer [2]. Also this test is conducted in a magnetic shielding case and thus the noise generated internally by the magnetometer can be verified. Selected screenshots of the spectrum analyzer measurements at $0.5 \mathrm{~Hz}$ and $1 \mathrm{~Hz}$ are shown in Figure 9. To specify the resolution, an MDS is represented by equation as follows;

$$
B=\mu_{0} n I=\mu_{0} n \frac{V}{R}
$$

where $\mu_{0}$ is the permeability of a sensor core, $n$ is turns of test solenoid per unit length and $I$ is an input current on a test solenoid. The resolution and noise level at selected frequencies are presented in Table 2.
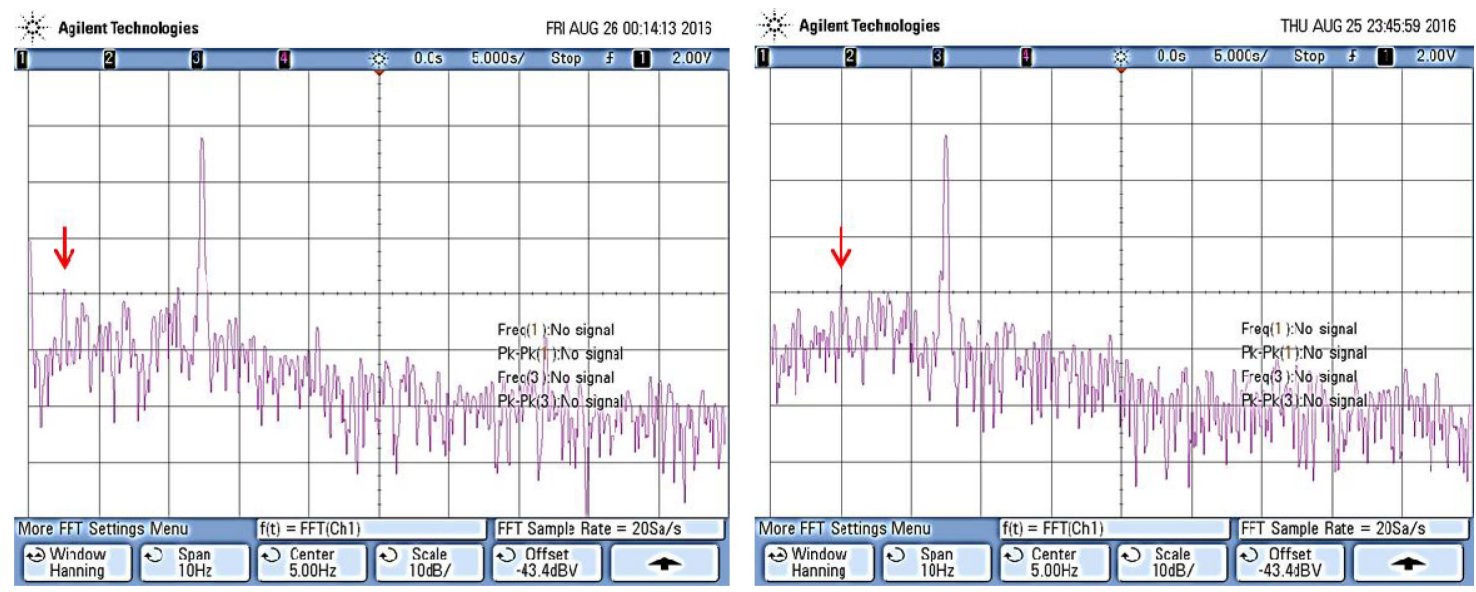

Fig. 9. (Color online) Screenshots of spectrum analyzer show exact responses of the search-coil magnetometer at $0.5 \mathrm{~Hz}$ (left) and $1 \mathrm{~Hz}$ (right) which is supplied by test solenoid frequency. Red arrows indicate spectral peak at the test frequency and it is $\sim 3 \mathrm{~dB}$ above the noise level. 
Table 2. Resolution and noise level of the SCM.

\begin{tabular}{cccccccc}
\hline \hline Sensor (axis) & $\mathrm{X}$ & $\mathrm{Y}$ & $\mathrm{X}$ & $\mathrm{Y}$ & $\mathrm{X}$ & $\mathrm{Y}$ \\
\hline Frequency $(\mathrm{Hz})$ & \multicolumn{2}{c}{0.5} & \multicolumn{4}{c}{1} & \multicolumn{2}{c}{2} \\
Noise level $(p T / \sqrt{\mathrm{Hz}})$ & 0.06 & 0.07 & 0.06 & 0.07 & 0.06 & 0.07 \\
Resolution $(p T / \sqrt{\mathrm{Hz}})$ & 0.08 & 0.1 & 0.08 & 0.1 & 0.09 & 0.1 \\
\hline
\end{tabular}

\subsection{Field Test}

The overall performance of the magnetometer has been examined at a field site in preparation for installation at the Jang Bogo Antarctic Station. The test site is currently used for an Astronomical Observatory in Bohyun Mountain, South Korea (BOAO, 36.1 N, 128.6 E, geographic) in which electromagnetic disturbance due to power lines and human activity is known to be minimal. A single axis magnetic sensor is installed approximately $200 \mathrm{~m}$ away from the observatory building and buried under the ground. The sensor is oriented along the local geomagnetic northsouth direction. Both frequency responses over sweeping frequencies and long-term ( $\sim 38$ hours) operation test have been conducted. The frequency response test is done using the external test solenoid as described in Section 3.1. Figure 10 shows the frequency response test in the field, showing the resonance frequency and cut-off frequency of the $\mathrm{SCM}$ at $5 \mathrm{~Hz}$ and $6 \mathrm{~Hz}$, respectively, which are based on the initial design of frequency range of the SCM.

Figure 11 shows the data obtained during the long-term field test starting at $\sim 10: 00$ UT on April 25, 2016. The top panel shows the observation data (output voltages over time) and bottom panel spectrogram (Fourier

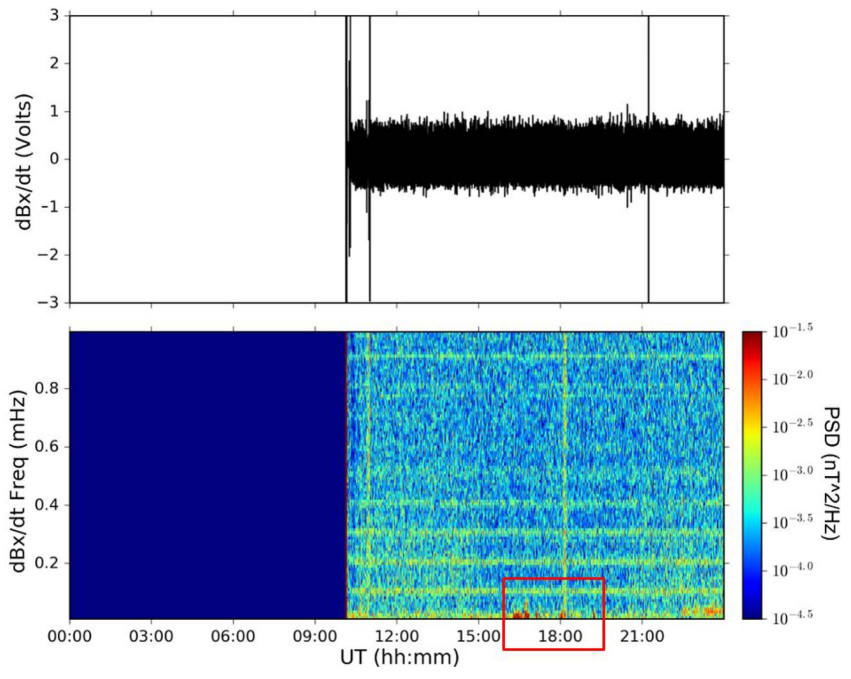

Fig. 11. (Color online) Time-series plot and spectrogram during the field test. The ULF Pi2 pulsation event is in the red rectangular box.

Transform power spectra over time). Based on the power spectral density (PSD) shown in the spectrogram, PSD of $<10^{-2.5} \mathrm{nT}^{2} / \mathrm{Hz}$ is considered to be external noise. Disturbances between 16:00 and 17:30 UT are found to be a ULF wave event. A blow-up image of the spectrogram is presented in Fig. 12, displaying a band-limited wave activity over the frequency range between 10 and 60 $\mathrm{mHz}$. For comparison, fluxgate magnetometer data from Mineyama, Japan (35.6 N, 135.1 E, L = 1.25) [15], whose location is similar to BOAO in terms of its geomagnetic latitude and local time, are presented on the bottom of the spectrogram in Fig. 12. Considering the sudden increases

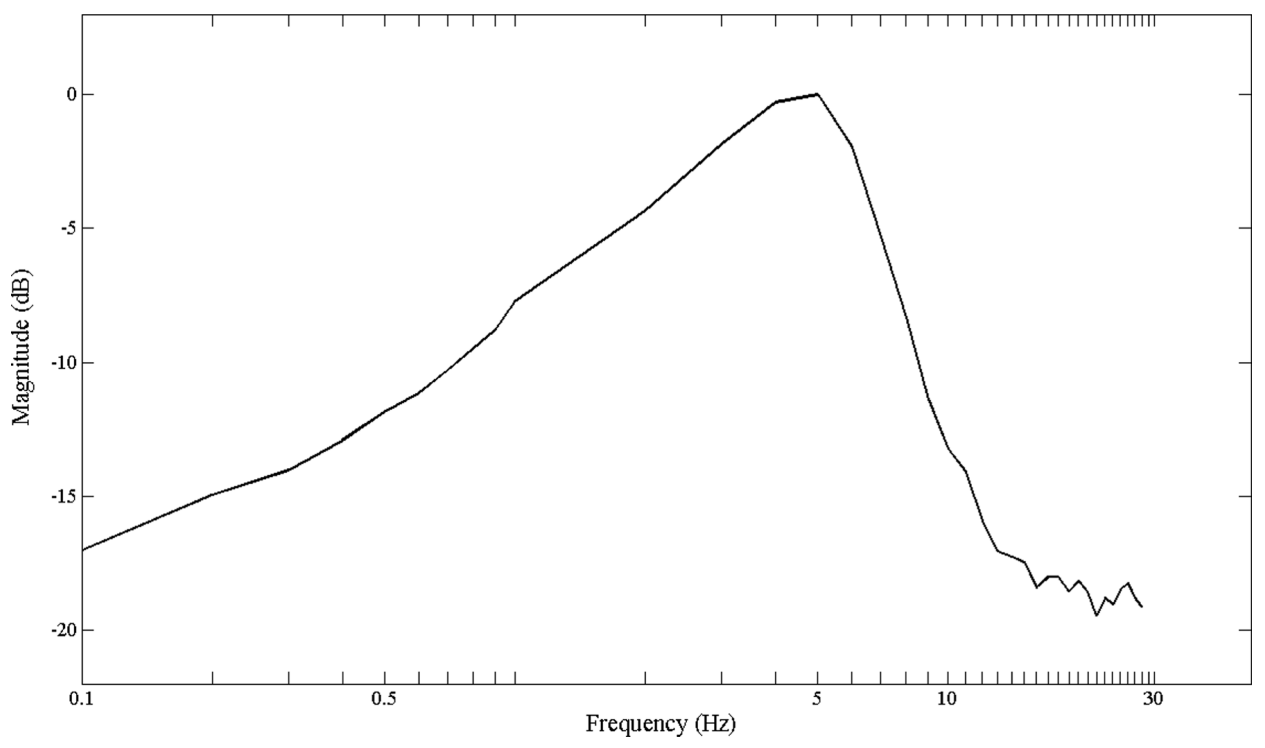

Fig. 10. Frequency response of the SCM over the frequencies from 0.1 to $30 \mathrm{~Hz}$ obtained during the field test at BOAO, showing the resonance frequency and cut-off frequency at $\sim 5 \mathrm{~Hz}$ and $\sim 6 \mathrm{~Hz}$, respectively (initial design of frequency range). 

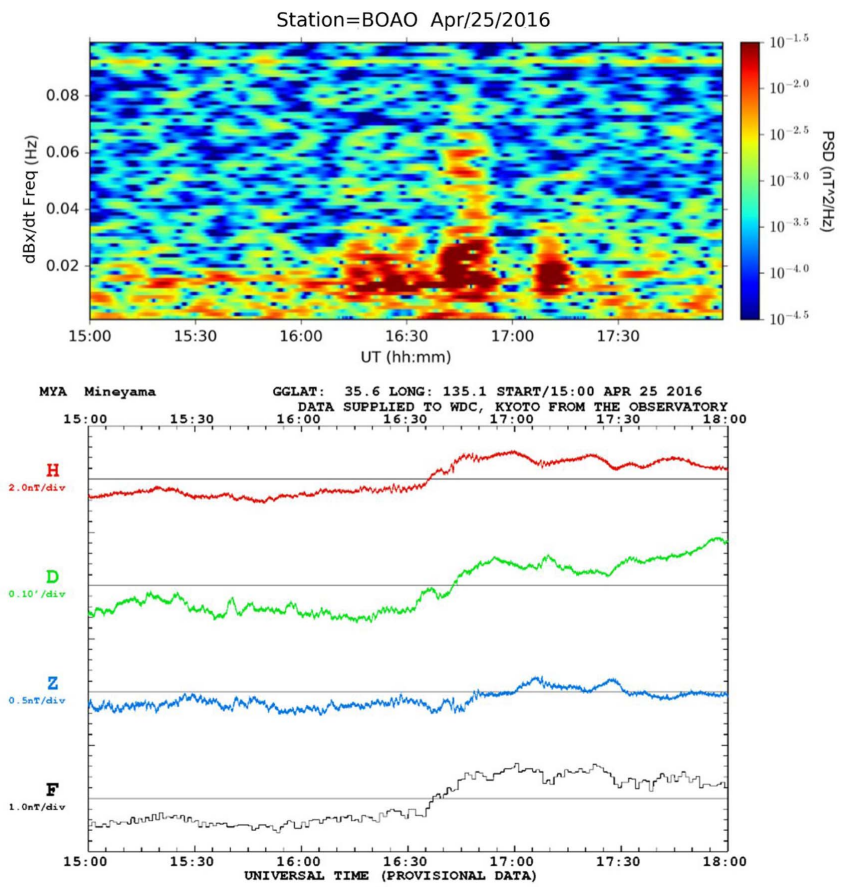

Fig. 12. (Color online) Spectrogram during the field test (top) and fluxgate magnetometer data at Mineyama site, Japan shows also $\mathrm{Pi} 2$ pulsations associated with a substorm activity (bottom).

in the fluxgate magnetometer data and magnetic local time (the magnetic local midnight that an observer on earth's surface back toward the sun is 16 UT at BOAO), the event is highly likely to be $\mathrm{Pi} 2$ pulsations associated with a substorm activity.

\section{Conclusions}

This paper describes development of a ground-based biaxial search-coil magnetometer for measurements of two vector components of magnetic field waves in the ULF range. For observations of ULF waves which play an important role in plasma dynamics in geospace, the SCM is designed to measure waves over the frequencies from 1 $\mathrm{mHz}$ to $5 \mathrm{~Hz}$ at the rate of $10 \mathrm{samples} / \mathrm{s}$. The performance has been fully examined to demonstrate a resolution of $0.1 p T / \sqrt{H z} @ 1 \mathrm{~Hz}$, which very much satisfies the requirement for observations of ULF waves on the ground. During a field test, ULF Pi2 pulsations associated with a substorm activity was detected, also showing its appropriate performance as a ground-based magnetometer for space research.

In an effort to augment ULF wave observation capability in Antarctica, KHU developed the magnetometer in collaboration with UNH. The instrument will be installed at the new Korean Antarctic station (Jang Bogo), which is operated by the KOPRI, in the austral summer 20162017. The Jang Bogo station is typically located in the magnetosphere cusp region where geomagnetic fields are not closed and thus the fields are directly linked to the Interplanetary Magnetic Field (IMF). In addition, the geomagnetic latitude of the station is similar to the United States McMurdo Station, which is expected to provide useful information about longitudinal extent of wave activities.

\section{Acknowledgment}

Development of the SCM was supported by the Korea Polar Research Institute (KOPRI) as the research project named "Study of magnetosphere-polar ionosphere-upper atmosphere coupling". We thank search-coil magnetometer project teams at New Jersey Institute of Technology and University of New Hampshire. We also acknowledge support from Bohyunsan Astronomical Observatory for the field test. Fluxgate magnetometer data from Mineyama are obtained from World Data Center for Geomagnetism, Kyoto.

\section{References}

[1] C. Coillot and P. Leroy, Induction Magnetometers: Principle, Modelling and ways of improvement, Magnetic Sensors-Principles and Applications, InTech, (2012) pp. 45-48.

[2] Hyomin Kim, Development of Ground-Based SearchCoil Magnetometer Systems in the Polar Regions and Studies of Ulf PC 1--2 Wave Propagation in the Ionospheric Waveg, Proquest (2010).

[3] L. Harang, Terr. Magn. and Atm. Electr. 41, 329 (1936).

[4] R. R. Heacock, J. Geophys. Res. 72, 3905 (1967).

[5] J. A. Jacobs, Y. Kato, S. Matsushita, and V. A. Troitskaya, J. Geophys. Res. 69, 180 (1964).

[6] James Lenz and Alan S. Edelstein, IEEE Sensors Journal 6, 631 (2006).

[7] J. Kangas, A. Guglielmi, and O. Pokhotelov, Space Sci. Rev. 83, 435 (1998).

[8] J. E. Lenz, Proceedings of the IEEE 78, 973 (1990).

[9] Pavel Ripka, Magnetic Sensors and Magnetometers, Artech House (2001) pp. 57-64.

[10] B. Stewart, Phil. Trans. R. Soc. Lond. 151, 423 (1861).

[11] E. Sucksdorff, Terr. Magn. and Atm. Electr. 41, 337 (1936).

[12] Séran \& Fergeaua, Review of Scientific Instruments 76, (2005) pp. 1-2.

[13] K. Takahashi, S. Ohtani, and B. J. Anderson, J. Geophys. Res. 100, 21929 (1995).

[14] S. Tumanski, Meas. Sci. Technol. 18, 35 (2007).

[15] World Data Center for Geomagnetism, Kyoto, Geomagnetic Data Service, http://wdc.kugi.kyoto-u.ac.jp/ 